\title{
The Predictive Value of Myeloperoxidase for Contrast-Induced Nephropathy After Percutaneous Coronary Intervention in Patients with Acute Myocardial Infarction
}

\section{Gaoliang Yan \\ Chengchun Tang \\ Genshan $\mathrm{Ma}$}

Department of Cardiology, Zhongda Hospital of Southeast University Medical School, Nanjing, 210009, People's Republic of China
Correspondence: Gaoliang Yan;

Genshan Ma

Department of Cardiology, Zhongda

Hospital of Southeast University

Medical School, No. 87 Dingjiaqiao,

Gulou District, Nanjing, 210009 ,

People's Republic of China

$\mathrm{Tel} / \mathrm{Fax}+862583262413$;

+862583262410

Emailyan_seu@hotmail.com;

magenshan@hotmail.com
Background: Higher serum myeloperoxidase (MPO) in patients with acute coronary syndrome is associated with adverse cardiovascular outcomes. Contrast-induced nephropathy (CIN) is associated with worse prognosis in patients with coronary artery disease following angiography. We have no idea whether patients with higher serum myeloperoxidase have a higher risk of CIN in acute myocardial infarction (AMI) after percutaneous coronary intervention (PCI).

Methods: This study involved 436 consecutive patients with AMI who had received PCI. Serum MPO levels were determined using enzyme-linked immunosorbent assay before administration of contrast media. Multivariate logistic regression analysis was used to analyze the independent risk factors for CIN after univariate analysis. The receiver operator characteristic (ROC) analysis was performed to evaluate the predictive value of MPO for CIN.

Results: Among the 436 patients, 79 individuals (18.1\%) suffered CIN after the PCI procedure. Patients who developed CIN had significantly higher MPO levels compared to those who did not ([203.8 (150.6-276.2)] versus [138.5 (129.9-149.2)]; $p<0.001)$. Multivariate logistic regression analysis revealed that MPO level (OR 1.023, 95\% CI: 1.017-1.029, $p<0.001)$ was an independent risk factor for the incidence of CIN after adjusting for the baseline information, blood indicators and angiography procedural parameters. The area under the ROC curve for predicting CIN of MPO was 0.848, and the optimum cutoff point of MPO was $147.38 \mathrm{ug} / \mathrm{L}$; the sensitivity and specificity were $82.3 \%$ and $72.3 \%$, respectively.

Conclusion: The results show that MPO is independently associated with an increased risk of CIN with AMI patients undergoing PCI. Further studies are needed to verify these results. Keywords: myocardial infarction, contrast-induced nephropathy, myeloperoxidase, percutaneous coronary intervention

\section{Introduction}

Contrast-induced nephropathy (CIN) is a common complication experienced by coronary artery disease patients after percutaneous coronary intervention (PCI). ${ }^{1}$ CIN negatively affects patients' prognosis, such as prolonging duration of hospitalization and increasing incidence of mortality and end-stage renal disease. ${ }^{2}$ However, there is no specific and effective treatment for CIN at present, although 
early identification and intervention can effectively improve the prognosis of patients at higher risk for CIN to some degree. At present, the clinical use of serum creatinine ( $\mathrm{SCr}$ ) level changes to diagnose $\mathrm{CIN}$ has some hysteresis. ${ }^{3}$ Hence, it is crucial to find some new and valuable predictors for predicting CIN.

As a member of the heme peroxidase superfamily, released by activated neutrophils, myeloperoxidase (MPO) has powerful pro-oxidative and pro-inflammatory properties. $^{4}$ Nitric oxide decreased after MPO was activated, ${ }^{5}$ leading to endothelial dysfunction. ${ }^{6}$ Recently, MPO has been recognized as an indicator of instability of plaque and patients with acute coronary syndrome admitted to emergency department because of chest pain are found to have higher MPO level. ${ }^{4-10}$ Furthermore, increased MPO is not likely to be specific to cardiac diseases; rather, it has also been associated with a variety of clinical conditions, including the development of atherosclerosis, ${ }^{4}$ together with numerous other inflammatory conditions, ${ }^{11}$ neurodegenerative disease, ${ }^{12}$ lung disorders ${ }^{13,14}$ and cancer, ${ }^{15}$ as activation of neutrophils and macrophages can occur in any infectious and inflammatory conditions.

Considering the strong relation between MPO and inflammation and oxidative stress, we speculated that leukocyte activation and, especially, MPO secretion both play a preeminent role in the initiation and extension phases of CIN. ${ }^{16}$ In this study, we assessed whether patients with high serum MPO levels are more likely to suffer CIN. We aimed to evaluate whether serum MPO can be used as an early diagnostic marker of CIN onset after PCI in acute myocardial infarction (AMI) patients.

\section{Patients and Methods Study Population}

This was a prospective and observational study (see Figure 1). Between January 2016 and September 2018, 436 patients with AMI who were treated by PCI in the Department of Cardiology, Zhongda Hospital Affiliated to Southeast University were selected. The criteria for admission were: (1) the diagnosis of ST-segment elevation myocardial infarction was in accordance with the 2015 guidelines $^{17}$ for the diagnosis and treatment of acute ST-segment elevation myocardial infarction of the Cardiovascular Diseases Branch of the Chinese Medical Association; and (2) the diagnosis of non-acute STsegment elevation myocardial infarction ${ }^{18}$ was in accordance with the guidelines for the diagnosis and treatment of non-ST-segment elevation acute coronary syndrome formulated by the Cardiovascular Diseases Branch of the Chinese Medical Association. The exclusion criteria were: (1) allergic history of iodine or iodine contrast agents; (2) patients with chronic renal insufficiency stage 5 or maintenance of hemodialysis/peritoneal dialysis; (3) CT, MRI and other contrast agents were performed within two weeks before admission; imaging examinations using other contrast agents were expected during the study period (except for PCI after one week), (4) nephrotoxic drugs had been used in the past two weeks (including high-dose loop diuretics, non-steroidal anti-inflammatory drugs, aminoglycosides, amphotericin B, proprietary Chinese medicines containing aristolochic acid, etc.); and (5) pateints with inflammatory diseases, autoimmune diseases, liver dysfunction, thyroid dysfunction, malignant tumors or infectious diseases. The research registration number was ChiCTR1900020824, and this study was approved by the Zhongda Hospital Ethics Committee; all patients provided informed consent.

\section{MPO Assay and Blood Analysis}

The blood samples for MPO assay were collected at baseline coronary angiography from the sheath of the radial/ femoral artery. Next, the concentration of serum myeloperoxidase (MPO) in patients was determined using a commercial enzyme-linked immunosorbent assay (ELISA) kit (produced by Beijing Union Rock Biotechnology Co. Ltd). In the microporous plate coated with anti-MPO antibody, the standard substance and the plasma to be tested were added, followed by the anti-MPO antibody with horseradish peroxidase to form a "sandwich" antigen-antibody complex, which catalyzed the conversion of the substrate TMB into a blue substance that turned yellow after termination. The depth of the color was proportional to the concentration of MPO antigen in the sample. The absorbance (OD) was measured at $450 \mathrm{~nm}$ by ELISA. The concentration of the analyte was calculated according to the $\mathrm{OD}$ value. $\mathrm{SCr}$ concentrations were measured before PCI, $48 \mathrm{~h}$ and $72 \mathrm{~h}$ after coronary angiography. CIN is currently defined either as an acute decrease in renal function after contrast exposure without evidence of other causes or as an $\mathrm{SCr}$ increase of $0.5 \mathrm{mg} /$ dL or a $25 \%$ increase compared to the baseline $\mathrm{SCr}$ level within 48 to $72 \mathrm{~h}$ following contrast media administration. ${ }^{19}$ 


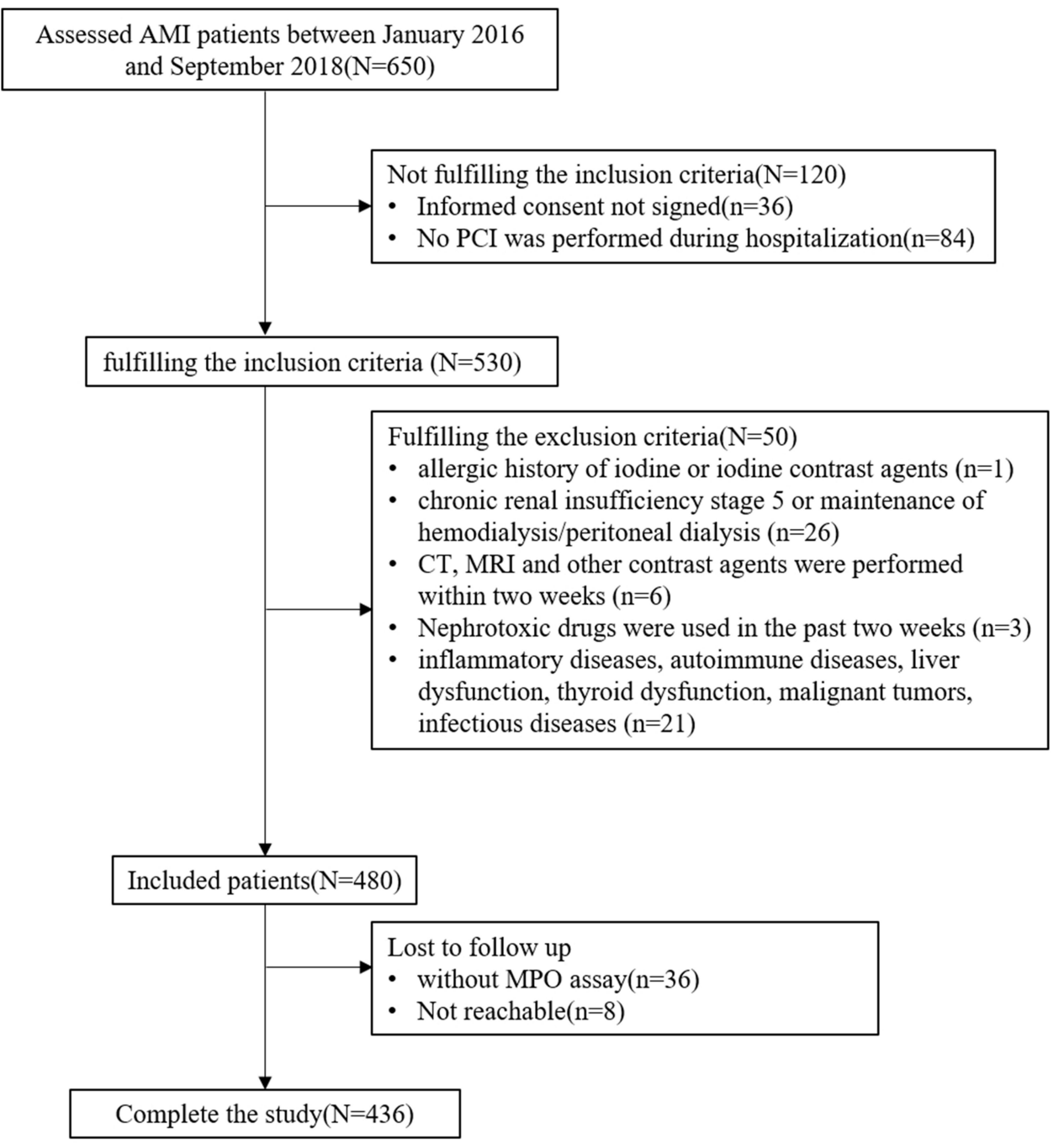

Figure I Study inclusion and exclusion criteria.

\section{Angiographic Definitions}

All patients underwent coronary angiography, and each coronary angiogram was preceded by experienced interventional cardiology using the femoral or radial approach. PCI of diseased vessels was conducted on the basis of coronary angiography findings. In principle, emergency PCI only deals with criminals' vessels. All patients were treated with load-dose double antiplatelet aggregation drugs (aspirin 300mg and clopidogrel $300 \mathrm{mg}$, or ticagrelor $180 \mathrm{mg}$ ) before surgery; Iodixanol (320 mg iodine $/ \mathrm{mL}$, GE Healthcare, Piscataway, New Jersey), a kind of isoosmolar, nonionic contrast agent was administered during all procedures. Antiplatelet drugs and statins were used after surgery; use of vasoactive drugs (such as dopamine, sodium nitroprusside, etc.), intra-aortic balloon pump, platelet membrane glycoprotein II b/III a receptor antagonists, and the use of $\beta$-receptor blockers, angiotensin-converting enzyme inhibitors(ACEIs) or angiotensin receptor blockers (ARBs), nitrates and calcium antagonists was determined by physicians based on respective clinical conditions. During the study period, nephrotoxic drugs (including large doses of loop diuretics, non-steroidal antiinflammatory drugs other than aspirin, aminoglycosides, amphotericin B and traditional Chinese medicine containing aristolochic acid, etc.) were avoided.

\section{Statistical Analysis}

SPSS version 19.0 software (SPSS Inc., Chicago, Illinois) was used for statistical analysis of data. The classified variables are expressed in frequencies and percentages, and compared by using $\chi^{2}$ and Fisher exact tests. The measurement data were expressed by mean + 
standard deviation or median and interquartile range, and comparisons between groups were expressed by independent sample $t$-test or Mann-Whitney $U$-test, where appropriate. Independent risk factors that might affect the occurrence of CIN in univariate analysis were filtered using multiple binary logistic regression analysis. The receiver operating characteristic (ROC) curve and the corresponding area under curve (AUC) were used to find out the best cutoff values of MPO for predicting CIN. Two-sided $p$-values of $<0.05$ were considered statistically significant.

\section{Results}

The study cohort included 436 consecutive patients who underwent PCI. Mean age was $62.9 \pm 12.7$ years [range: 26-92]), and 335 (76.8\%) were male. Baseline creatinine was $86.3 \pm 41.6 \mathrm{umol} / \mathrm{L}$; consistently, average GFR was $64.4 \pm 24.5 \mathrm{~mL} / \mathrm{min} / 1.73 \mathrm{~m}^{2}$. Of these pateints, 116 (26.6\%) had diabetes mellitus, 242 (55.5\%) had hypertension and $35(8.0 \%)$ had received previous percutaneous coronary intervention. Overall, 79 patients (18.1\%) developed CIN after the PCI procedure.

Baseline clinical characteristics of the study population are summarized in Table 1. Elderly patients were more likely to suffer from CIN. Patients with hypertension, diabetes mellitus, old myocardial infarction, and prior PCI on admission $(p<0.05)$ had a higher incidence of CIN. There were no significant differences between the groups regarding gender, body mass index, smoking, systolic blood pressure, stroke, LVEF, prevalence of STEMI or in-hospital medications $(p>0.05)$.

Comparisons between the laboratory parameters of the two groups are shown in Table 2. Patients who developed CIN have significantly higher MPO levels at baseline compared to non-CIN patients: ([203.8 (150.6-276.2)] versus $[138.5(129.9-149.2)] ; p<0.001)$. In addition, patients in the CIN group had significantly higher baseline WBC and creatinine levels than those in the non-CIN group $(p<0.05)$. However, patients in the CIN group showed significantly lower levels of lymphocyte and monocyte than patients in the non-CIN group $(p<0.05)$.

Table 3 presents angiography and procedural characteristics of the two groups. The patients with CIN had higher rates of aspiration and emergency PCI. However, no significant differences were found in any other procedural characteristics, such as contrast agent volume, culprit vessel, number of vessels or rates of thrombus among the groups. Also, the proportion of stents implanted and the
Table I Baseline Clinical Characteristics Between Patients with $\mathrm{CIN}$ and without CIN

\begin{tabular}{|c|c|c|c|}
\hline Variables & $\begin{array}{l}\text { CIN } \\
(n=79)\end{array}$ & $\begin{array}{l}\text { Non-CIN } \\
(n=357)\end{array}$ & $p$-value \\
\hline Age (years) & $66.4 \pm 12.0$ & $62.1 \pm 12.7$ & 0.006 \\
\hline Male, n (\%) & $55(69.6)$ & $280(78.4)$ & 0.105 \\
\hline BMI $\left(\mathrm{kg} / \mathrm{m}^{2}\right)$ & $25.0 \pm 3.6$ & $24.9 \pm 3.6$ & 0.820 \\
\hline Systolic BP (mmHg) & $128 \pm 22.6$ & $|29 \pm 2| . \mid$ & 0.755 \\
\hline Smoking, n (\%) & $34(54.0)$ & $178(63.6)$ & 0.196 \\
\hline Hypertension, n (\%) & $55(69.6)$ & $187(52.4)$ & 0.006 \\
\hline Diabetes mellitus, $n$ (\%) & $37(46.8)$ & $79(22.1)$ & $<0.001$ \\
\hline OMI, n (\%) & $8(10.1)$ & $7(2.0)$ & 0.002 \\
\hline Pre-PCI, n (\%) & $14(17.7)$ & $21(4.8)$ & 0.002 \\
\hline Stroke, n (\%) & $3(3.8)$ & $3(0.8)$ & 0.076 \\
\hline $\mathrm{LV}(\mathrm{cm})$ & $4.77 \pm|.5|$ & $5.0 I \pm 2.31$ & 0.366 \\
\hline LVEF (\%) & $45(36-55)$ & $50(40-61)$ & 0.142 \\
\hline Cardiogenic shock, n (\%) & $7(8.9)$ & $23(6.4)$ & 0.461 \\
\hline Acute heart failure, $\mathrm{n}(\%)$ & $10(12.7)$ & $34(9.5)$ & 0.410 \\
\hline Diagnosis, n (\%) & & & 0.814 \\
\hline NSTEMI & $52(65.8)$ & $230(64.4)$ & \\
\hline STEMI & $27(34.2)$ & $127(35.6)$ & \\
\hline \multicolumn{4}{|l|}{ Medication, n (\%) } \\
\hline Aspirin & $77(97.5)$ & $34 I(95.5)$ & 0.753 \\
\hline$\beta$-blocker & $60(75.9)$ & $277(77.6)$ & 0.767 \\
\hline ACEI & $25(31.6)$ & I46(40.9) & 0.128 \\
\hline ARB & $19(24.1)$ & $79(22.1)$ & 0.711 \\
\hline $\mathrm{CCB}$ & $19(24.1)$ & $83(23.2)$ & 0.879 \\
\hline Statins & $77(97.5)$ & $343(96.1)$ & 0.748 \\
\hline Loop diuretics & $27(34.2)$ & $125(35.0)$ & $>0.99$ \\
\hline Spironolactone & $27(34.2)$ & $123(34.5)$ & $>0.99$ \\
\hline
\end{tabular}

Note: Data are presented as $n$ (\%) or median (25th-75th percentile).

Abbreviations: BMI, body mass index; BP, blood pressure; Pre-PCl, prepercutaneous coronary intervention; LV, left ventricular; LVEF, left ventricular ejection fraction; NSTEMI, non-ST-elevation myocardial infarction; STEMI, ST-elevation myocardial infarction; ACEl, angiotensin-converting enzyme inhibitor; ARB, angiotensin receptor blocker; $\mathrm{CCB}$, calcium channel blocker.

number and length of stents were similar between patients with CIN and those without CIN ( $p>0.05)$.

Obviously, MPO level is significantly linked to the incidence of CIN in the univariate model. Age, hypertension, diabetes, old myocardial infarction, emergency PCI and aspiration were also positively associated with the development of CIN. Multivariate logistic regression analysis revealed that baseline MPO level (OR 1.023, 95\% CI: $1.017-1.029, p<0.001$ ), age (OR 1.030, 95\% CI: $1.002-1.059, p=0.034)$, presence of hypertension (OR 2.336, 95\% CI: 1.161-4.700, $p=0.017$ ), diabetes (OR 2.037, 95\% CI: 1.027-4.041, $p=0.042$ ), OMI (OR 9.787, 95\% CI: $2.402-39.882, p=0.001)$ and WBC level 
Table 2 Baseline Laboratory Data Between Patients with CIN and without CIN

\begin{tabular}{|c|c|c|c|}
\hline Variables & $\begin{array}{l}\text { CIN } \\
(n=79)\end{array}$ & $\begin{array}{l}\text { Non-CIN } \\
(n=357)\end{array}$ & $\begin{array}{l}p- \\
\text { value }\end{array}$ \\
\hline MPO (ug/L) & $\begin{array}{l}203.8 \\
(150.6-276.2)\end{array}$ & $\begin{array}{l}\mid 38.5 \\
(|29.9-| 49.2)\end{array}$ & $<0.001$ \\
\hline WBC $\left(\times 10^{9} / \mathrm{L}\right)$ & $11.2 \pm 3.4$ & $10.4 \pm 3.7$ & 0.073 \\
\hline Hemoglobin $(g / L)$ & $134.3 \pm 21.6$ & $138.4 \pm 20.1$ & 0.113 \\
\hline PLT $\left(\times 10^{9} / \mathrm{L}\right)$ & $216.2 \pm 69.4$ & $214.4 \pm 61.9$ & 0.818 \\
\hline Lymphocyte $\left(\times 10^{9} / \mathrm{L}\right)$ & $1.5 \pm 1.1$ & $1.7 \pm 1.1$ & 0.076 \\
\hline Monocyte $\left(\times 10^{9} / \mathrm{L}\right)$ & $0.45 \pm 0.27$ & $0.49 \pm 0.24$ & 0.118 \\
\hline Pre-creatinine $(\mu \mathrm{mol} / \mathrm{L})$ & $91.7 \pm 40.5$ & $79.0 \pm 45.0$ & 0.017 \\
\hline eGFR & $57.8 \pm 11.2$ & $69.8 \pm 16.2$ & $<0.001$ \\
\hline Uric acid (mmol/L) & $322.4 \pm 112.6$ & $330.4 \pm 94.4$ & 0.521 \\
\hline Urea nitrogen $(\mathrm{mmol} / \mathrm{L})$ & $5.7 \pm 3.2$ & $6.9 \pm 4.5$ & 0.546 \\
\hline $\mathrm{TC}(\mathrm{mmol} / \mathrm{L})$ & $4.8 \pm 2.5$ & $4.6 \pm 1.2$ & 0.510 \\
\hline $\mathrm{TG}(\mathrm{mmol} / \mathrm{L})$ & $1.9 \pm 1.5$ & $2.2 \pm 1.7$ & 0.705 \\
\hline LDL-C (mmol/L) & $2.8 \pm 0.7$ & $2.9 \pm 1.1$ & 0.563 \\
\hline HDL-C(mmol/L) & $1.1 \pm 0.3$ & $I . I \pm 0.4$ & 0.717 \\
\hline Troponin I(ng/mL) & $\begin{array}{l}7.26 \\
(0.42-20)\end{array}$ & $9.08(0.12-26)$ & 0.426 \\
\hline CK-MB (ng/mL) & $12.8(2.6-23)$ & I8.4 (2.0-27) & 0.166 \\
\hline FBG (mmol/L) & $8.8 \pm 4.0$ & $8.7 \pm 4.2$ & 0.944 \\
\hline
\end{tabular}

Note: Data are presented as median (25th-75th percentile).

Abbreviations: MPO, myeloperoxidase; WBC, white blood count; PLT, platelet counts; eGFR, estimated glomerular filtration rate; TC, total cholesterol; TG, triglyceride; LDL-C, low-density lipoprotein-cholesterol; HDL-C, high-density lipoprotein-cholesterol; CK-MB, creatine kinase-MB; FBG, fasting blood glucose.
Table 3 Baseline Angiographic Data Between Patients with CIN and without CIN

\begin{tabular}{|c|c|c|c|}
\hline Variables & $\begin{array}{l}\text { CIN } \\
(n=79)\end{array}$ & $\begin{array}{l}\text { Non-CIN } \\
(n=357)\end{array}$ & $\begin{array}{l}p- \\
\text { value }\end{array}$ \\
\hline Emergency $\mathrm{PCl}, \mathrm{n}(\%)$ & $67(84.8)$ & $257(72.0)$ & 0.022 \\
\hline IABP, n (\%) & $5(6.3)$ & $19(5.3)$ & 0.784 \\
\hline Contrast dose, $\mathrm{mL}$ & $\begin{array}{l}100 \\
(100-120)\end{array}$ & $100(100-120)$ & 0.180 \\
\hline Culprit vessel, n (\%) & & & 0.889 \\
\hline Left main & $\mathrm{I}(\mathrm{I} .3)$ & $4(1.1)$ & \\
\hline $\begin{array}{l}\text { Left anterior } \\
\text { descending }\end{array}$ & $4 I(5 I .9)$ & 174(48.7) & \\
\hline Left circumflex & $\mathrm{II}(13.9)$ & $37(10.4)$ & \\
\hline Right coronary artery & $24(36.4)$ & $13 \mid(36.7)$ & \\
\hline $\begin{array}{l}\text { Number of vessels, } \\
\mathrm{n}(\%)\end{array}$ & & & 0.341 \\
\hline I & $13(16.5)$ & $55(15.4)$ & \\
\hline 2 & $14(17.7)$ & $91(25.5)$ & \\
\hline 3 & $52(65.8)$ & $2 I I(59.1)$ & \\
\hline Thrombus n (\%) & $58(73.4)$ & $227(63.6)$ & 0.117 \\
\hline Aspiration, n (\%) & $58(73.4)$ & $218(61.1)$ & 0.040 \\
\hline Stenting, n (\%) & $66(83.5)$ & $321(89.9)$ & 0.116 \\
\hline Number of stents, $n$ & $0.95 \pm 0.53$ & $1.01 \pm 0.47$ & 0.305 \\
\hline Pre-expansion, n (\%) & $25(31.6)$ & $151(42.3)$ & 0.099 \\
\hline Length of stents, $\mathrm{cm}$ & $22.7 \pm \mid 4.1$ & $25.3 \pm 14.1$ & 0.138 \\
\hline
\end{tabular}

Note: Data are presented as $n$ (\%) or median (25th-75th percentile). Abbreviations: $\mathrm{PCI}$, percutaneous coronary intervention; IABP, intra-aortic balloon pump.

to be the protective factor for CIN after adjusting for the baseline data, blood parameter and angiography procedural parameters (Table 4).
(OR 1.116, 95\% CI: 1.022-1.217, $p=0.014)$ were all independent risk factors. However, the level of eGFR (OR $0.211,95 \%$ CI: $0.142-0.421, p=0.003$ ) was shown

Table 4 Univariate and Multivariate Logistic Regression Analysis of CIN Risk Factors

\begin{tabular}{|l|c|c|c|c|c|c|}
\hline \multirow{2}{*}{} & \multicolumn{3}{|c|}{ Univariate Analysis } & \multicolumn{3}{c|}{ Multivariate Analysis } \\
\cline { 2 - 7 } & OR & $95 \%$ Cl & $\mathbf{P}$ & OR & $95 \%$ CI & P \\
\hline MPO & 1.021 & $1.016-1.027$ & $<0.001$ & 1.023 & $1.017-1.029$ & $<0.001$ \\
Age & 1.028 & $1.008-1.049$ & 0.007 & 1.030 & $1.002-1.059$ & 0.034 \\
Male & 0.630 & $0.367-1.083$ & 0.095 & 0.871 & $0.412-1.839$ & 0.716 \\
Hypertension & 2.083 & $1.235-3.513$ & 0.006 & 2.336 & $1.161-4.700$ & 0.017 \\
Diabetes mellitus & 3.100 & $1.866-5.150$ & $<0.001$ & 2.037 & $1.027-4.041$ & 0.042 \\
OMI & 5.634 & $1.979-16.035$ & 0.001 & 9.787 & $2.402-39.88$ & 0.001 \\
Pre-PCI & 3.446 & $1.667-7.126$ & 0.001 & 1.993 & $0.676-5.874$ & 0.211 \\
WBC & 1.060 & $0.994-1.19$ & 0.074 & 1.116 & $1.022-1.217$ & 0.014 \\
Lymphocyte & 0.785 & $0.600-1.027$ & 0.078 & 0.847 & $0.412-1.839$ & 0.716 \\
eGFR & 0.271 & $0.181-0.568$ & $<0.001$ & 0.211 & $0.142-0.421$ & 0.003 \\
Emergency & 2.173 & $1.127-4.188$ & 0.021 & 2.458 & $0.909-6.644$ & 0.076 \\
Aspiration & 1.761 & $1.024-3.030$ & 0.041 & 1.266 & $0.556-2.883$ & 0.575 \\
\hline
\end{tabular}

Abbreviations: MPO, myeloperoxidase; OMI, old myocardial infarction; Pre-PCl, pre-percutaneous coronary intervention; WBC, white blood count; eGFR, estimated glomerular filtration rate. 


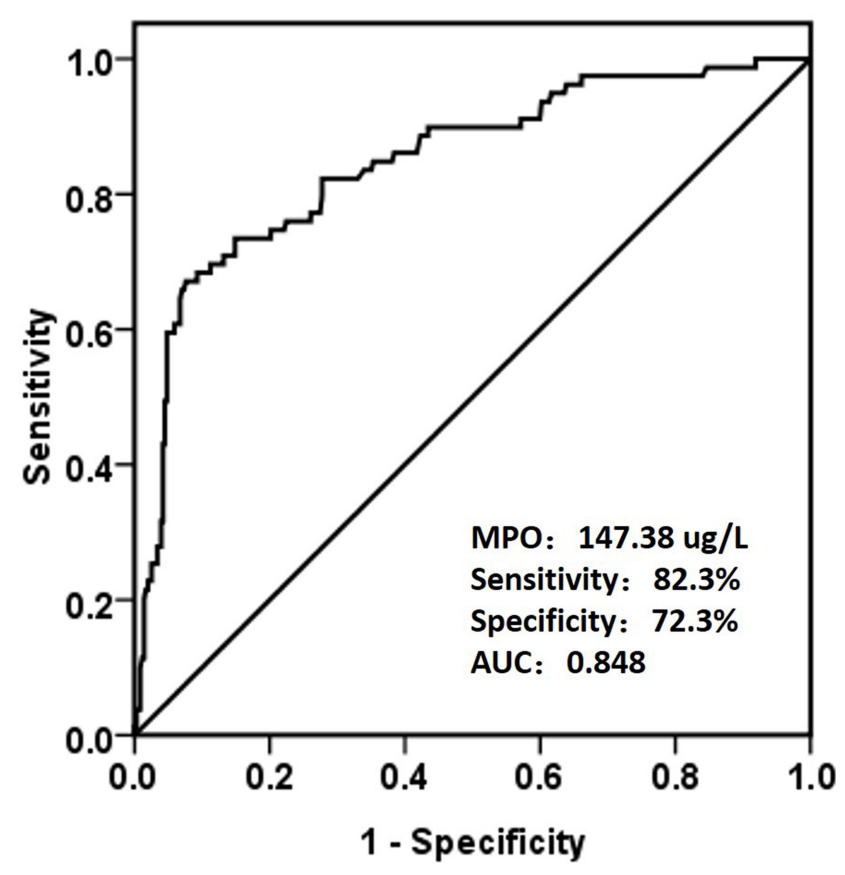

Figure 2 Receiver operating characteristic curve of the MPO level in predicting CIN. The optimum cutoff point of MPO was 147.38 ug/L, with a sensitivity of $82.3 \%$ and a specificity of $72.3 \%$.

According to the ROC curve analysis (Figure 2), the MPO level was an accurate predictor for the development of CIN; the AUC was 0.848 for the baseline MPO level (95\% CI: $0.797-0.898, p<0.001$ ). The optimum cutoff point for MPO was $147.38 \mathrm{ug} / \mathrm{L}$, with a sensitivity of
$82.3 \%$ and a specificity of $72.3 \%$. Furthermore, we found that the incidence of CIN in the high MPO group (39.6\%; $\mathrm{MPO}>147.38 \mathrm{ug} / \mathrm{L})$ was significantly higher $(p<0.001$; Figure 3) compared to the group without elevated MPO $(5.1 \%, \mathrm{MPO}<147.38 \mathrm{ug} / \mathrm{L})$.

\section{Discussion}

This study found that CIN was a common complication after PCI in AMI patients. This is consistent with previous reports $^{20,21}$ in which the incidence of CIN in patients with AMI after PCI was still as high as $18.1 \%$. In the present study, we evaluated the predictive value of MPO for risk of CIN in AMI patients undergoing PCI. Our data, the first report in the literature on this subject, suggested that high baseline MPO level is a pre-procedural predictor of CIN. Age, hypertension, diabetes mellitus, old myocardial infarction and baseline WBC are also independent predictors of CIN in such patients.

The precise pathophysiologic mechanisms of CIN have not been fully elucidated yet. Possible mechanisms include renal vasoconstriction, decrease of renal blood flow, formation of reactive oxygen species, medullary hypoxia and direct cytotoxicity. ${ }^{22-25}$ In view of the similarity between many mechanisms, such as inflammation and oxidative stress induced by MPO with the pathogenesis of CIN, we speculate that there is an inevitable correlation between the occurrence of CIN and the level of

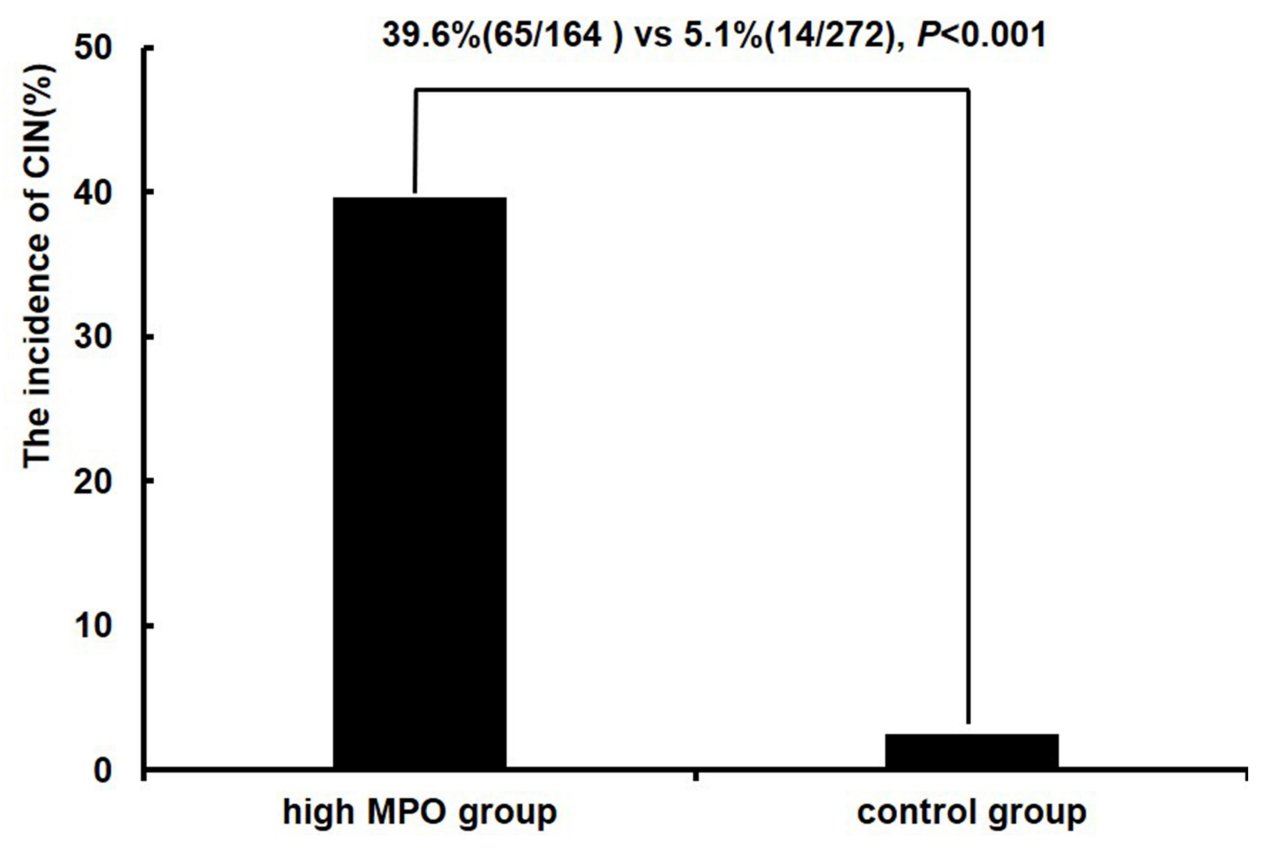

Figure $3 \mathrm{CIN}$ incidence in different groups. 
MPO. Here, we firstly reported that baseline MPO level was independently correlated with risk of CIN in AMI patients undergoing PCI. With respect to the mechanisms underlying this association, The MPO-hydrogen peroxidechloride system resulted in a variety of chlorinated protein and lipid adducts, which can lead to kidney injury. ${ }^{26}$ The inflammation induced and exacerbated by MPO antibody complexes in necrotizing glomerulonephritis following MPO is a crucial pathogenic factor in glomerular and tubulointerstitial diseases in hyperlipidemia-induced renal damage in rodents. ${ }^{27}$ MPO is involved in the adherence of neutrophils to the glomerular basement membrane and the degradation of the basement membrane by oxidants at sites of attachment. Johnson et $\mathrm{al}^{28}$ revealed that MPO-mediated glomerular disease gives rise to glomerular morphologic changes, endothelial and mesangial cell injury, activation of platelets and subsequent proliferative responses mimicking inflammatory and proliferative glomerulonephritis in humans. In particular, the interaction of MPO with nitric oxide metabolism makes the actions of oxidants more complex and may explain the bimodal salubrious and unwholesome effects of the MPOhydrogen peroxide-chloride system in redox-modulated renal diseases. ${ }^{29}$ 3-nitrotyrosine, lipid hydroperoxides and other resultant oxidation products accumulate in the fibrotic kidneys and the plasma of chronic renal insufficiency patients, indicating oxidative stress aggravated during the progression of kidney disease. ${ }^{30}$ Therefore, we propose a hypothesis that MPO and MPO-derived oxidants may participate as mediators of oxidative modification of biomolecules/tissues in the development of CIN Moreover, the multivariate logistic regression analysis in this study revealed that age, hypertension, diabetes mellitus, old myocardial infarction and baseline WBC were also independent predictors of CIN in such patients in accordance with the literature. ${ }^{31,32}$ Patients with OMI often simultaneously suffer from a variety of other medical and cardiovascular diseases with the progress of ventricular remodeling after myocardial infarction; many patients have cardiac and renal insufficiency and the level of inflammatory cytokines increases significantly, which is often accompanied by the activation of inflammatory cells. Yamamoto et al found, through multivariate analysis, that high preoperative WBC count was a novel risk factor for developing CIN following mechanical thrombectomyan. ${ }^{33}$ Similar reports show an increase in inflammation-related molecules in CIN after PCI, including WBC counts (especially neutrophils). ${ }^{34}$ Inflammatory cells cause the release of oxygen free radicals, vasoconstrictors, leukotrienes and thromboxane, leading to kidney damage. If the white blood cell count is high before surgery, for various reasons, the increase of systemic inflammatory reaction may promote the pathological progress of CIN.

\section{Limitations}

This study has some limitations that should be considered. First, this is an observational study, the number of patients studied with CIN is relatively small, and all participants were recruited from a single center. Second, our data only detects the baseline MPO level; dynamic observations on the changes of MPO and SCr were not conducted. Third, the measurements of inflammatory markers and other relevant biomarkers, ${ }^{35}$ such as neutrophil gelatinase-associated lipocalin, urinary interleukin 18 and kidney injury molecule-1, are not available, thus study of the inflammatory and oxidative stress mechanisms of CIN is not deep enough. Thus, we could not dig out the essential mechanisms between serum MPO levels and deteriorated renal injury.

\section{Conclusion}

In conclusion, our study demonstrates that a higher preprocedural MPO level is a risk factor for the development of CIN in patients with AMI. However, larger sample and prospective randomized controlled trials are needed to evaluate and confirm whether high preoperative MPO levels affect CIN or lowering MPO treatment reduces the incidence of CIN.

\section{Data Sharing Statement}

Data related to this manuscript can be made available from the corresponding author upon reasonable request.

\section{Ethics Approval and Consent to Participate}

This study was conducted with approval from the Ethics Committee of Zhongda Hospital, Southeast University Medical School. This study was conducted in accordance with the Declaration of Helsinki. Written informed consent was obtained from all participants.

\section{Acknowledgments}

This study was supported by a grant from the National Natural Science Foundation of China (81600227) and Jiangsu Provincial Key Medical Discipline (Laboratory 
ZDXKA2016023). These funding agencies had no influence on the study design, data collection or analysis, decision to publish or preparation of the article.

\section{Disclosure}

The authors declare that they have no competing interests concerning this paper.

\section{References}

1. Nash K, Hafeez A, Hou S. Hospital-acquired renal insufficiency. Am J Kidney Dis. 2002;39(5):930-936. doi:10.1053/ajkd.2002.32766

2. James MT, Ghali WA, Tonelli M, et al. Acute kidney injury following coronary angiography is associated with a long-term decline in kidney function. Kidney Int. 2010;78(8):803-809. doi:10.1038/ ki.2010.258

3. Shaker OG, El-Shehaby A, El-Khatib M. Early diagnostic markers for contrast nephropathy in patients undergoing coronary angiography. Angiology. 2010;61(8):731-736. doi:10.1177/ 0003319710373093

4. Nicholls SJ, Hazen SL. Myeloperoxidase and cardiovascular disease. Arterioscler Thromb Vasc Biol. 2005;25(6):1102-1111. doi:10.1161/ 01.ATV.0000163262.83456.6d

5. Abu-Soud HM, Hazen SL. Nitric oxide is a physiological substrate for mammalian peroxidases. $J$ Biol Chem. 2000;275 (48):37524-37532. doi:10.1074/jbc.275.48.37524

6. Vita JA, Brennan ML, Gokce N, et al. Serum myeloperoxidase levels independently predict endothelial dysfunction in humans. Circulation. 2004;110(9):1134-1139. doi:10.1161/01. CIR.0000140262.20831.8F

7. Delporte C, Van Antwerpen P, Vanhamme L, Roumeguere T, Zouaoui Boudjeltia K. Low-density lipoprotein modified by myeloperoxidase in inflammatory pathways and clinical studies. Mediators Inflamm. 2013;2013:971579. doi:10.1155/2013/971579

8. Mocatta TJ, Pilbrow AP, Cameron VA, et al. Plasma concentrations of myeloperoxidase predict mortality after myocardial infarction. $J$ Am Coll Cardiol. 2007;49(20):1993-2000. doi:10.1016/j. jacc.2007.02.040

9. Yunoki K, Naruko T, Komatsu R, et al. Relation of elevated levels of plasma myeloperoxidase to impaired myocardial microcirculation after reperfusion in patients with acute myocardial infarction. $\mathrm{Am}$ J Cardiol. 2010;105(7):922-929. doi:10.1016/j.amjcard.2009.11.013

10. Brennan ML, Penn MS, Van Lente F, et al. Prognostic value of myeloperoxidase in patients with chest pain. $N$ Engl $J$ Med. 2003;349(17):1595-1604. doi:10.1056/NEJMoa035003

11. Hoy A, Leininger-Muller B, Kutter D, Siest G, Visvikis S. Growing significance of myeloperoxidase in non-infectious diseases. Clin Chem Lab Med. 2002;40(1):2-8. doi:10.1515/CCLM.2002.002

12. Yap YW, Whiteman M, Cheung NS. Chlorinative stress: an under appreciated mediator of neurodegeneration? Cell Signal. 2007;19 (2):219-228. doi:10.1016/j.cellsig.2006.06.013

13. Kettle AJ, Chan T, Osberg I, et al. Myeloperoxidase and protein oxidation in the airways of young children with cystic fibrosis. $\mathrm{Am}$ J Respir Crit Care Med. 2004;170(12):1317-1323. doi:10.1164/ rccm.200311-1516OC

14. Van Der Vliet A, Nguyen MN, Shigenaga MK, Eiserich JP, Marelich GP, Cross CE. Myeloperoxidase and protein oxidation in cystic fibrosis. Am J Physiol Lung Cell Mol Physiol. 2000;279(3): L537-546. doi:10.1152/ajplung.2000.279.3.L537

15. Atwal M, Lishman EL, Austin CA, Cowell IG. Myeloperoxidase enhances etoposide and mitoxantrone-mediated DNA damage: a target for myeloprotection in cancer chemotherapy. Mol Pharmacol. 2017;91(1):49-57. doi:10.1124/mol.116.106054
16. Akcay A, Nguyen Q, Edelstein CL. Mediators of inflammation in acute kidney injury. Mediators Inflamm. 2009;2009:137072. doi: $10.1155 / 2009 / 137072$

17. China Society of Cardiology of Chinese Medical Association EBoCJoC, China Society of Cardiology of Chinese Medical Association Editorial Board of Chinese Journal of C. [Guideline on the diagnosis and therapy of ST-segment elevation myocardial infarction]. Zhonghua Xin Xue Guan Bing Za Zhi. 2015;43 (5):380-393. Chinese.

18. Chinese Society of Cardiology of Chinese Medical A, Editorial Board of Chinese Journal of C. [Guideline of non-ST segment elevation acute coronary syndrome]. Zhonghua Xin Xue Guan Bing Za Zhi. 2012;40(5):353-367. Chinese.

19. Stacul F, van der Molen AJ, Reimer P, et al. Contrast induced nephropathy: updated ESUR Contrast Media Safety Committee guidelines. Eur Radiol. 2011;21(12):2527-2541. doi:10.1007/ s00330-011-2225-0

20. Tsai TT, Patel UD, Chang TI, et al. Contemporary incidence, predictors, and outcomes of acute kidney injury in patients undergoing percutaneous coronary interventions: insights from the NCDR Cath-PCI registry. JACC Cardiovasc Interv. 2014;7(1):1-9. doi:10.1016/j.jcin.2013.06.016

21. Narula A, Mehran R, Weisz G, et al. Contrast-induced acute kidney injury after primary percutaneous coronary intervention: results from the HORIZONS-AMI substudy. Eur Heart J. 2014;35 (23):1533-1540. doi:10.1093/eurheartj/ehu063

22. Pisani A, Riccio E, Andreucci M, et al. Role of reactive oxygen species in pathogenesis of radiocontrast-induced nephropathy. Biomed Res Int. 2013;2013:868321. doi:10.1155/2013/868321

23. Andreucci M, Faga T, Pisani A, Sabbatini M, Michael A. Acute kidney injury by radiographic contrast media: pathogenesis and prevention. Biomed Res Int. 2014;2014:362725.

24. Azzalini L, Spagnoli V, Ly HQ. Contrast-induced nephropathy: from pathophysiology to preventive strategies. Can J Cardiol. 2016;32 (2):247-255. doi:10.1016/j.cjca.2015.05.013

25. Kurtul A, Ornek E. Platelet to lymphocyte ratio in cardiovascular diseases: a systematic review. Angiology. 2019;70(9):802-818. doi:10.1177/0003319719845186

26. Kisic B, Miric D, Dragojevic I, Rasic J, Popovic L. Role of myeloperoxidase in patients with chronic kidney disease. Oxid Med Cell Longev. 2016;2016:1069743. doi:10.1155/2016/1069743

27. Hazen SL, Zhang R, Shen Z, et al. Formation of nitric oxide-derived oxidants by myeloperoxidase in monocytes: pathways for monocyte-mediated protein nitration and lipid peroxidation in vivo. Circ Res. 1999;85(10):950-958. doi:10.1161/01.RES.85.10.950

28. Johnson RJ, Couser WG, Chi EY, Adler S, Klebanoff SJ. New mechanism for glomerular injury. Myeloperoxidase-hydrogen peroxide-halide system. J Clin Invest. 1987;79(5):1379-1387. doi:10.1172/JCI112965

29. Malle E, Buch T, Grone HJ. Myeloperoxidase in kidney disease. Kidney Int. 2003;64(6):1956-1967. doi:10.1046/j.15231755.2003.00336.x

30. Himmelfarb J. Uremic toxicity, oxidative stress, and hemodialysis as renal replacement therapy. Semin Dial. 2009;22(6):636-643. doi:10.1111/j.1525-139X.2009.00659.x

31. Lin KY, Zheng WP, Bei WJ, et al. A novel risk score model for prediction of contrast-induced nephropathy after emergent percutaneous coronary intervention. Int $J$ Cardiol. 2017;230:402-412. doi:10.1016/j.ijcard.2016.12.095

32. Yin WJ, Yi YH, Guan XF, et al. Preprocedural prediction model for contrast-induced nephropathy patients. J Am Heart Assoc. 2017;6(2). doi:10.1161/JAHA.116.004498

33. Yamamoto Y, Yamamoto N, Kanematsu Y, et al. High white blood cell count is a risk factor for contrast-induced nephropathy following mechanical thrombectomy for acute ischemic stroke. Cerebrovasc Dis Extra. 2020;10(2):59-65. doi:10.1159/000507918 
34. Yuan Y, Qiu H, Hu X, et al. Predictive value of inflammatory factors on contrast-induced acute kidney injury in patients who underwent an emergency percutaneous coronary intervention. Clin Cardiol. 2017;40(9):719-725. doi:10.1002/clc.22722
35. Klein SJ, Brandtner AK, Lehner GF, et al. Biomarkers for prediction of renal replacement therapy in acute kidney injury: a systematic review and meta-analysis. Intensive Care Med. 2018;44(3):323-336. doi:10.1007/s00134-018-5126-8

\section{Publish your work in this journal}

The International Journal of General Medicine is an international, peer-reviewed open-access journal that focuses on general and internal medicine, pathogenesis, epidemiology, diagnosis, monitoring and treatment protocols. The journal is characterized by the rapid reporting of reviews, original research and clinical studies across all disease areas. The manuscript management system is completely online and includes a very quick and fair peer-review system, which is all easy to use. Visit http://www.dovepress.com/ testimonials.php to read real quotes from published authors.

Submit your manuscript here: https://www.dovepress.com/international-journal-of-general-medicine-journal 